The AstrophysiCAL Journal, 475:519-533, 1997 February 1

(C) 1997. The American Astronomical Society. All rights reserved. Printed in U.S.A.

\title{
EVOLUTION OF SPIRAL GALAXIES. VII. TIME EVOLUTION OF THE RADIAL DISTRIBUTIONS OF ABUNDANCES
}

\author{
Mercedes Mollá, Federico Ferrini, ${ }^{1}$ AND ANGeles I. DíAZ \\ Departamento de Física Teórica, Universidad Autónoma de Madrid, E-28049 Madrid, Spain \\ Received 1996 February 13; accepted 1996 August 26
}

\begin{abstract}
In this work, the time evolution of the radial distributions of elemental abundances in the disks of galaxies is analyzed; in particular, we investigate whether they become flatter, steeper, or remain equal with time. In order to do this, observational data related to elemental abundances and metallicity estimates are briefly reviewed. Most of them refer to our Galaxy, but when possible, other spiral galaxies' data have been included. We show how the traditional view of steeper abundance gradients at later times may be questionable and how recent observational data sets are not in contradiction with the opposite behavior.

Theoretical trends are deduced from the application of the multiphase model of chemical evolution, developed by Ferrini and collaborators and successfully used to describe the galactic components and different morphological type galaxies. In particular, we analyze the time evolution of the main properties of the following spiral galaxies: NGC 224, NGC 300, NGC 598, NGC 628, NGC 3198, NGC 6946, and the Milky Way.

We show that the radial distributions of elemental abundances may flatten with time if we assume isolated galaxy evolution. The rate for local metallicity enrichment varies with the galactocentric radius and the morphological type, being higher for the central regions and more intense for the early types. The flattening rate of abundance distributions is larger for these types of galaxies, while in the latest types the radial gradient remains almost constant with time.
\end{abstract}

Subject headings: galaxies: abundances — galaxies: evolution — galaxies: spiral

\section{INTRODUCTION}

A large number of observations from various groups and of different spiral galaxies has established that the abundances of elements decrease in the disks with increasing galactocentric radius (see, e.g., Díaz 1989). The rate of decrease inside each galaxy has been generally considered as constant, as a first approximation to the more complex elemental distributions and its value or radial gradient results different from galaxy to galaxy.

A few studies have attempted to correlate the values of abundance gradients and the absolute abundances of selected elements, such as oxygen, with the morphological types of galaxies (Vila-Costas \& Edmunds 1992; Zaritsky, Kennicutt, \& Huchra 1994). There is reasonable evidence that the abundance gradients are flatter for galaxies of early morphological types (Oey \& Kennicutt 1993), which also have higher absolute abundances.

A classical interpretation of the morphological classification of spiral galaxies considers this to be the effect of different star formation rates characterizing each type (Larson \& Tinsley 1978; Sandage 1986). In this way, the morphological sequence may be interpreted as evolutionary: early-type galaxies have evolved more rapidly than those of later types, as a result of a higher star formation rate at earlier times. The variations in time of a few quantities such as gas distributions can also be interpreted according to this scheme (Ferrini \& Galli 1988). We may imagine that similarly the abundance distributions can vary, according to the different star formation histories of galaxies.

The relationship between the abundance of a tracer

\footnotetext{
${ }^{1}$ Dipartimento di Fisica, Università di Pisa, Piazza Torricelli 2, 56100 Pisa, Italy.
}

element, such as iron relative to hydrogen, and time (the so-called age-metallicity relation, or AMR) has been studied by several authors in the solar neighborhood since Twarog (1980). The same analysis has been extended to other Galactic regions: the halo over the solar neighborhood (Schuster $\&$ Nissen 1989) and the outer disk (Geisler 1987). The rate of enrichment in the different regions has not been the same, since it depends on the history of star formation, which has not been uniform over the Galaxy. Therefore there is no reason to believe that the radial distributions of elements should have always been the same as presently observed. Connecting this argument with the previous one, the idea that the abundance distributions vary in time as a consequence of the star formation history in each galaxy seems worthy of inquiry.

The comprehension of the radial abundance distributions appears to be related to many other aspects of galaxies, such as radial distributions of gas and mass and metal distributions in other regions, like halos and bulges, and eventually, it appears connected with the very process of galaxy formation, which is fundamental to give the imprint to the subsequent evolution.

The problem of the evolution of radial elemental distributions with time has not yet been adequately solved, neither from observations, since different data sets for old objects seem to indicate different solutions, nor from theoretical models, since, depending on the different involved assumptions (see, e.g., Tosi 1996), radial gradients result that are flatter or steeper at earlier times. This uncertainty motivates our work.

In the following, we present a critical collection of data for the Milky Way and other spiral galaxies $(\S 2)$; in $\S 3$, we analyze the results of our theoretical models and compare them to existing data for several galaxies and to other model predictions for the Milky Way. Finally, in $\S 4$ we 
discuss the consequences of the modeling and state our conclusions.

\section{OBSERVATIONS}

The information that is commonly used as constraints for theoretical chemical evolution models of galaxies refers to radial distributions of gas and total mass surface densities, star formation rate (SFR), and elemental abundances.

We shall distinguish between the present-day situation and the old population distributions. The denomination of young or old for a certain class of objects may be totally subjective: in the literature some objects are called old if their ages are greater than $1 \mathrm{Gyr}$, a time that appears rather short when compared to the characteristic evolutionary times of galaxies, which are of the order of the Hubble time. Furthermore, we must remember that the rate of growth of elemental abundances has been widely discussed in the literature; a reasonable convergence of typical timescales of enrichment of the order of 2-5 Gyr has been reached. Therefore, assuming an age of $13 \mathrm{Gyr}$ for all galaxies, in our case young refers to the last Gyr and old to $7 \mathrm{Gyr}$ ago and before.

An independent and accurate discussion of observations for the Milky Way can be found in Prantzos \& Aubert (1995); our agreement with these authors is almost complete.

\subsection{Today \\ 2.1.1. H I Distributions}

Different data about the radial distribution of atomic gas in the Milky Way are in reasonable agreement (see review by Rana 1991). Current estimates (see Sanders, Solomon, \& Scoville 1984; Güsten \& Mezger 1982; Wouterloot et al. 1990) including the correction for the self-absorption of the $21 \mathrm{~cm}$ line (Garwood \& Dickey 1989) show that H I increases from a surface density $\sigma_{\mathrm{H} \mathrm{II}}=3.5-4 M_{\odot} \mathrm{pc}^{-2}$ for $R=4 \mathrm{kpc}$ to $\sigma_{\mathrm{H} \mathrm{II}}=7-8 M_{\odot} \mathrm{pc}^{-2}$ for $R=12 \mathrm{kpc}$. Then it decreases to $\sigma_{\mathrm{H} \mathrm{II}}=5 M_{\odot} \mathrm{pc}^{-2}$ for $R=15 \mathrm{kpc}$.

For the disks of external galaxies, it has been possible to obtain $\mathrm{H}$ I radial distributions from $21 \mathrm{~cm}$ line observations, too. In general, they show a behavior similar to that observed in the Galactic disk, with a maximum at a given radius and a decrease for larger radii. This maximum occurs at a larger radius for earlier morphological types and moves toward the inner regions for the latest types. References for these distributions may be found in Mollá, Ferrini, \& Díaz (1996, hereafter MFD).

\subsection{2. $\mathrm{H}_{2}$ Distributions}

There are a large number of observations of the bright $\mathrm{CO}(1-0)$ emission line along the Galactocentric radius in the Milky Way (e.g., Talbot 1980; Güsten \& Mezger 1982; Sanders et al. 1984; Grabelsky et al. 1987; Bronfmann et al. 1988; Wouterloot et al. 1990), as in other external galaxies (see Young 1987). The molecular gas density is obtained from the $\mathrm{CO}$ line intensity through the conversion factor $\chi=N\left(\mathrm{H}_{2}\right) / \int T_{\mathrm{CO}} d v$. This factor is estimated from the virial masses of self-gravitating molecular clouds in the Milky Way, gamma-ray emissivity, and optically thin molecular lines and dust extinction (see Kenney 1995). Most determinations are in the range $(2-8) \times 10^{20}$ molecules $\mathrm{cm}^{-2}(\mathrm{~K}$ $\left.\mathrm{km} \mathrm{s}^{-1}\right)^{-1}$, with a "standard value" of $3.2 \pm 0.5$. This factor is not constant with the Galactocentric distance in the Milky Way: it is lower for the inner regions of the
Galactic disk and higher for the outer ones (Mead \& Kutner 1988; Mead, Kutner, \& Evans 1990; Sodroski 1991). This may be explained as due to radial variations of abundances: in the presence of a larger number of carbon atoms, the $\mathrm{CO}-\mathrm{H}_{2}$ interactions may increase, with the same amount of $\mathrm{H}_{2}$. Consequently, $\chi$ may be also different from galaxy to galaxy, since they vary in metallicity and show different radial abundance gradients. There is compelling evidence in this direction: Deul \& den Hartog (1990) made estimates of the molecular gas mass in the galaxy NGC 598 (M33), where individual molecular clouds were resolved, and found that this mass is a factor of 3 larger than the value estimated from the $\mathrm{CO}$ luminosity using a Galactic conversion factor. Recently, Verter \& Hodge (1995) have claimed that the conversion factor $\chi$ increases by a factor of 3 when the oxygen abundance drops by a factor of 6 . In good agreement with these authors, Wilson (1995) analyzed this metallicity dependence, using data on the virial masses of molecular clouds for some nearby galaxies. She shows that, indeed, $\chi$ increases up to a factor of 4-5 when the oxygen abundance decreases by a factor of 10 . If this calibration is applied, the radial distributions of molecular gas surface densities flatten as compared to the old ones estimated using a constant $\chi$-factor.

We have hence used a calibration dependent on the metallicity as described in Tosi \& Díaz (1985), by obtaining a factor $\chi(R)$ in every galaxy; the references to the distributions used are in MFD. The resulting radial distributions for the $\mathrm{H}_{2}$ surface density are very similar to those resulting from the calibration by Wilson.

\subsubsection{SFR Distributions}

Present SFRs are more difficult to obtain. For the Milky Way, it has been estimated by some authors using different methods (see Rana 1991; Prantzos \& Aubert 1995). Radial distributions of the SFR surface density exist for a few external galaxies. They are usually derived from the $\mathrm{H} \alpha$ recombination line, related to the Lyman continuum photons emitted by young massive stars. The sample have been enlarged using Wilson \& Scoville's (1991) relation between $\mathrm{H} \alpha$ luminosity and the SFR, and the $\mathrm{H} \alpha$ fluxes compiled by Kennicutt (1989).

\subsubsection{Abundance Distributions}

Radial abundance distributions for oxygen and nitrogen, estimated from observations of $\mathrm{H}$ II regions, constitute the commonly used information to constrain chemical evolution models (see Ferrini et al. 1994, hereinafter FMPD; MFD). As we explained in our previous paper about external galaxies, difficulties in the determination of abundances introduce a bias in the derivation of the radial distribution of abundances toward the outer part of the disk, with the inner region being severely undersampled. The data show radial gradients for most galaxies observed (see, e.g., Díaz 1989); abundances are higher for inner regions and lower for the outer ones, and the value of this difference is larger for galaxies of the later types.

For the Milky Way the traditional value of the radial gradient of oxygen abundance has been $-0.081 \pm 0.017$ dex $\mathrm{kpc}^{-1}$, based on observations from Shaver et al. (1983) of $\mathrm{H}$ II regions. Subsequent sets of observations have changed this estimate somewhat.

The initial observations of $\mathrm{H}$ II regions were limited to a short range of Galactocentric distances. If we complement the sample of Shaver et al. with the recent estimates by 
Vilchez \& Esteban (1996), the oxygen radial gradient decreases to $-0.062 \pm 0.009$ dex $\mathrm{kpc}^{-1}$. Since oversolar oxygen abundances, i.e., $12+\log (\mathrm{O} / \mathrm{H})>9.1$, cannot be considered established for well-known calibration problems (see, e.g., Díaz 1994), the gradient calculated discarding the corresponding entries reduces to $-0.052 \pm 0.008 \mathrm{dex}$ $\mathrm{kpc}^{-1}$.

Luck (1982) discussed the abundance distributions for $G$ and $\mathrm{K}$ supergiants, revising previous abundance determinations. The resulting gradient of $[\mathrm{Fe} / \mathrm{H}]$, over a limited spatial range $(7.5 \mathrm{kpc}<R<10.5 \mathrm{kpc})$, was $-0.13 \pm 0.03$ dex $\mathrm{kpc}^{-1}$, but the author reminds the reader that the error represents only a formal uncertainty, and systematic corrections to the determined abundances and distances might affect the result.

From the data collection of Prantzos \& Aubert, we became aware of the importance of the paper by Kaufer et al. (1994) that summarizes recent work on abundances of young B stars. For these authors, the abundances derived from B stars are more reliable than those derived from $\mathrm{H}$ II regions (or planetary nebulae [PNs]). The result is that for B stars from 6 to $16 \mathrm{kpc}$ there is no detectable gradient for oxygen $\left(0.000 \pm 0.009 \mathrm{dex} \mathrm{kpc}^{-1}\right)$ and only a marginal gradient for nitrogen $\left(-0.026 \pm 0.009 \mathrm{dex} \mathrm{kpc}^{-1}\right)$. Data from Shaver et al. (1983) and from Fich \& Silkey (1991) for H II regions have a large intrinsic scatter, yielding a small gradient in oxygen $\left(-0.045 \pm 0.022 \mathrm{dex} \mathrm{kpc}^{-1}\right)$ and nitrogen $\left(-0.007 \pm 0.013 \mathrm{dex} \mathrm{kpc}^{-1}\right)$. The possibility of a larger gradient in the inner Galaxy cannot be excluded, but it is not in any way evident.

An interesting class of disk objects are the open clusters. As Janes and Friel have underlined several times (see, e.g., in Alfaro \& Delgado 1995) they are rather excellent tracers of the disk evolution. We have considered the sets of open clusters for which $[\mathrm{Fe} / \mathrm{H}]$, Galactocentric distance, and age have been estimated by Panagia \& Tosi (1981), Cameron (1985), and Friel (1995) together. The first two groups refer to young open clusters ( $\tau \lesssim 1 \mathrm{Gyr}$ ) while the last is a large homogeneous collection of data including older open clusters (see $\S 2.2 .2$ ). Considering the young objects all together (58 open clusters), we obtain a gradient of $-0.089 \pm 0.025$ dex $\mathrm{kpc}^{-1}$ over a radial range of $6.5-11 \mathrm{kpc}$.

The radial abundance distributions of external galaxies have been taken from the references given in Table 2 of MFD. They have been derived from observations of $\mathrm{H}$ II region emission lines. For most $\mathrm{H}$ II regions the data refer to oxygen, but in some cases abundances for other elements, mainly nitrogen and/or sulphur, are given.

\subsection{Yesterday \\ 2.2.1. Age-Metallicity Relations}

The AMR, established by Twarog (1980) and later confirmed by other authors (Carlberg et al. 1985; Barry 1988) for the supernova region, and the AMR derived for the outer regions of the disk $(R>8 \mathrm{kpc})$ by Geisler (1987), could be used to reconstruct information about the radial gradients at early times. From the curves fitted to Barry's, Twarog's, and Geisler's data, it is impossible to determine the difference between the corresponding abundances and hence to estimate the variation with time of the radial gradient. The best fits have to be done with too few points, and this difference fluctuates. Edvardsson et al. (1993) have demonstrated that an intrinsic scatter in the AMR is unavoidable. In the fourth paper of this series (Pardi, Ferrini, \& Matteucci 1995), it was shown how the large scatter in Edvardsson et al.'s (1993) data can be understood in terms of the overlapping of stellar populations belonging to the various phases of gas accumulation on the disk.

\subsubsection{Old Object Abundances and Metallicities}

Accurate determinations of age, distance, and abundances for extended sets of objects in the Galactic disk are difficult to obtain. To compare data from the interstellar medium and evolved stars, it is necessary to assume that the estimated abundances are the same as those of the interstellar gas out of which they were formed. To determine radial distributions at past times, there are old objects meeting the above requirement, such as Cepheids, globular or old open clusters, planetary nebulae, and old stars, for which abundances have been estimated. In most cases, these data refer to metallicity defined by the iron abundance relative to the Sun, $[\mathrm{Fe} / \mathrm{H}]$.

It is worthwhile to mention the fact that it may be misleading to derive oxygen abundances from knowledge of iron abundance, as is often done in the literature (see Ferrini 1995 for a discussion of this point). The use of an "empirical" linear relationship of $[\mathrm{O} / \mathrm{Fe}]$ versus $[\mathrm{Fe} / \mathrm{H}]$ for regions different from those where it has been determined may be arbitrary and produce systematic errors: oxygen and iron are synthesized at different rates (Matteucci \& Greggio 1986), according to the history of a certain region and the connected ones, e.g., a ring in the disk and the overhanging halo. The relationship of $[\mathrm{O} / \mathrm{Fe}]$ versus $[\mathrm{Fe} / \mathrm{H}]$ is not unique in the Galaxy, and may also be different in external galaxies, so therefore we do not consider the oxygen abundances estimated with this method to be reliable. This conclusion is supported not only by theoretical arguments (see FMPD, Fig. 1c), but also by observational results: see in Edvardsson et al. (1993) Figures 22a-22c, where the authors present their observed $[\mathrm{O} / \mathrm{Fe}]$ versus $[\mathrm{Fe} / \mathrm{H}]$ relations for different ranges of Galactocentric distance in the Milky Way. Nobody would seriously try to fit linear regression lines to these three distributions, although a reasonable linear fit could be imposed on the cumulative set, Figure 15a. It is evident that, looking for behaviors depending on location, it may be misleading to average over space the observations and deduce correlations to be applied everywhere.

Harris (1981) made one of these first analyses for old objects using type II Cepheid stars, estimating their metallicities from the luminosity-period relations. According to this author, the radial gradient was flat at early times. It may be noted that Harris's set presents a typical problem: the value of the gradient is strongly dependent on the width of the interval of Galactocentric distances over which a linear fit is searched, and the distances and metallicities of Cepheids, according to Grenon (1988), are highly uncertain.

Zinn (1985), and later Alfaro, Cabrera-Caño, \& Delgado (1993) for the subsample of rich globular clusters, reached a similar conclusion, of a small radial gradient for an old population. In their works, there is a clear bias because the disk globular clusters were selected using a metallicity criterion: all globular clusters having $[\mathrm{Fe} / \mathrm{H}] \leq-0.8$ were eliminated from the disk population. When looking for abundance distributions of different populations, the selection must be made according to the kinematic properties; hence if objects with a height over the Galactic disk $z \leq 1$ 
kpc and with a rotation velocity $v_{\text {rot }} \geq 80 \mathrm{~km} \mathrm{~s}^{-1}$ are selected, a radial gradient of $-0.156 \pm 0.094 \mathrm{dex} \mathrm{kpc}^{-1}$ is obtained. If, futhermore, the clusters with Galactocentric distance $R<2 \mathrm{kpc}$ are eliminated, in order to account for any possible bulge contamination, a value of $-0.273 \pm 0.103 \mathrm{dex} \mathrm{kpc}^{-1}$ is found. Even correcting following Köppen (1994) for the vertical gradient $\left(-0.13 \pm 0.056 \operatorname{dex~} \mathrm{kpc}^{-1}\right)$, we find a value of $-0.148 \pm 0.094 \mathrm{dex} \mathrm{kpc}^{-1}$ with all data and a gradient of $-0.261 \pm 0.102 \mathrm{dex} \mathrm{kpc}^{-1}$ for $R>2 \mathrm{kpc}$, both significantly steeper than the one suggested by Köppen: $-0.01 \pm 0.03$ dex $\mathrm{kpc}^{-1}$; this clearly shows that great care must be taken in analyzing and interpreting the data. Regarding external galaxies, data have been obtained for the globular cluster system in NGC 224 (M31) (Huchra, Brodie, \& Kent 1991). The problem to obtain an unbiased gradient is the same: the selection has to be made using kinematic criteria. If we use a rotational velocity greater than $80 \mathrm{~km} \mathrm{~s}^{-1}$ to select the disk globular clusters, we obtain a gradient of $-0.051 \pm 0.040 \mathrm{dex} \mathrm{kpc}^{-1}$ for this galaxy. On the other hand, to use globular clusters properties to reconstruct the old disk structure may be risky: it is not clearly established whether the globular clusters are members of the halo or the disk populations; contaminations are possible (see, e.g., Minniti 1996, who attributes some globular clusters to the bulge).

Lewis \& Freeman (1989) analyzed a K-giant sample; their abundances, which have a scatter of \pm 0.5 dex, do not show any gradient. It is impossible to discriminate distinct aged populations in their sample, since they ascribed all their objects to a unique disk population aged more than 1 Gyr. Moreover, their distance determinations are not very reliable.

Type II PNs are interesting objects to study radial gradients since they have intermediate-mass progenitors and small chemical enrichment. Maciel \& Köppen (1994) revised the situation and estimated an oxygen gradient of $-0.069 \pm 0.006 \mathrm{dex} \mathrm{kpc}{ }^{-1}$ for 91 objects, in the radial range $5 \mathrm{kpc}<R<11.5 \mathrm{kpc}$. Type II PNs are not very old objects in our discussion, since the typical progenitor mass may be around $2 M_{\odot}$, with a mean life of $\sim 1.7 \mathrm{Gyr}$. We note that the conclusion of Maciel \& Köppen that the radial gradient for the oxygen abundance has been almost the same for the last $5 \mathrm{Gyr}$ is an extrapolation: from PNs one may deduce that the oxygen abundance gradient has not varied very much in a certain time range. Futhermore, if the time range for PNs is 1.5-5 Gyr and the radial distances of the observed ones range from 5 to $11.5 \mathrm{kpc}$, this can be easily understood in terms of the AMR (see Fig. 5), which has a limited growth in those intervals.

From the old open clusters in the catalog of Friel (1995), we obtain an iron gradient $d[\mathrm{Fe} / \mathrm{H}] / d R-0.082 \pm 0.017$ dex kpc ${ }^{-1}$ for all objects (40); if we distinguish according to her morphological age index (MAI) to order temporally the clusters, we obtain for intermediate-age clusters $(1<\tau<3)$ $-0.072 \pm 0.020 \mathrm{dex} \mathrm{kpc}^{-1}$ and for older clusters $(3<\tau)$ $-0.115 \pm 0.037 \mathrm{dex} \mathrm{kpc}^{-1}$, which indicates that the older open cluster distribution presents a steeper gradient. If we consider together the clusters from Cameron, Panagia \& Tosi, and Friel, divided into two bins of age (or MAI) below and above 3, we find a tendency toward a flattening in the distribution of younger open clusters for Galactocentric radii less than $9 \mathrm{kpc}$ : for $\tau<3$ in the range $7.3 \mathrm{kpc}<R<9$

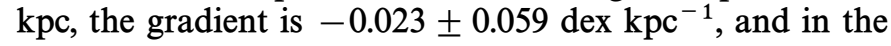

range $9 \mathrm{kpc}<R<14 \mathrm{kpc}$, the gradient is $-0.083 \pm 0.027$ dex $\mathrm{kpc}^{-1}$.

A rich sample of $F$ and $G$ dwarf stars has been observed by Edvardsson et al. (1993); the authors make estimates of metallicities, Galactocentric distances, maximum heights over the galactic plane, and orbital eccentricities. We have analyzed these data to look for any indication of the evolution of abundance gradients with time. We selected a subsample for the disk population using the two criteria recommended by the authors: the eccentricity must be $\epsilon \leq 0.16$, and the maximum height over the disk must be $z \leq 0.26 \mathrm{kpc}$. We then divided the sample into two bins with different ages, scaling the age of the sample stars to our adopted Galactic age of 13 Gyr. The resulting oldest objects, with $\tau \geq 10.0 \mathrm{Gyr}$, have been excluded because of the high uncertainty involved in their age determination $(\geq 2.5 \mathrm{Gyr})$. We have then calculated the radial iron gradient for every bin, finding, for $\tau<3.2 \mathrm{Gyr},-0.072 \pm 0.054$ dex $\mathrm{kpc}^{-1}$ (61 stars) and, for $\tau>3.2 \mathrm{Gyr},-0.149 \pm 0.064$ dex $\mathrm{kpc}^{-1}$ (41 stars). Prantzos \& Aubert (1995) found the same value for the young stars of this sample, but a different value for the older stars, since they did not select according to eccentricity and height above the disk.

More recently, Jønch-Sørensen (1995) presented a new set of observations of 435 faint $F$ stars in the Galactic disk, with Galactocentric distances in the range $2-12 \mathrm{kpc}$, in a study aimed to shed light on the evolution of abundance gradients. The author finds his results to be in "conflict with the current understanding of the processes responsible for the slope and the evolution of the gradient in the disk." First, plotting all the objects together, considering only a selection on the vertical distance to the plane, he finds a cloud of points with a dispersion in $[\mathrm{Fe} / \mathrm{H}]$ of about \pm 0.5 , from which the radial gradient is not significantly different from zero, a result very similar to what Lewis \& Freeman (1989) found (Figs. 16a, 16b). After that, since JønchSørensen arranges the objects temporally, he can investigate the "aging effect on the gradients" (see his Fig. 17). The results are as follows: (1) for $R<8 \mathrm{kpc}$, the abundance of $[\mathrm{Fe} / \mathrm{H}]$ is almost constant in time $(\sim 0.00 \pm 0.02 \mathrm{dex}$ $\mathrm{kpc}^{-1}$ ); (2) for $R>8 \mathrm{kpc}$, the older age bins have a steeper gradient (about -0.150 or $-0.200 \mathrm{dex} \mathrm{kpc}^{-1}$ ) with respect to the younger bins, which are quite flatter.

\subsection{The Aging of Gradients}

The analysis of existent data leads us to the following conclusions (Table 1): from Kaufer et al. there is evidence for an almost zero gradient for oxygen in young populations (B stars and $\mathrm{H}$ II regions); this is also supported by recent data from Vílchez \& Esteban (1996) on H II regions in the outer disk. For nitrogen there is evidence (Kaufer et al. 1994) of a very small gradient for the same young populations. From type II PNs, older objects, Maciel \& Köppen (1994) obtained about $-0.07 \mathrm{dex} \mathrm{kpc}^{-1}$.

For iron, we can distinguish between young and old populations:

For $\tau<3$, we have data for open clusters and $\mathrm{F}$ and $\mathrm{G}$ stars, and all the authors quoted above agree on a rather low value for this gradient, not sensibly different from 0.00 for Jønch-Sørensen, roughly $-0.07 \mathrm{dex} \mathrm{kpc}^{-1}$ for Edvardsson et al., and around -0.02 and -0.08 , respectively, for the inner and outer Galaxy for Friel, Panagia \& Tosi, and Cameron. 
TABLE 1

ObSERVED ABUndANCE GRADIENTS

\begin{tabular}{|c|c|c|c|c|c|c|}
\hline \multirow[b]{2}{*}{ ELEMENT } & \multicolumn{3}{|c|}{ POPULATIONS $^{\mathrm{a}}$} & \multirow{2}{*}{$\begin{array}{c}\text { RANGE } \\
(\mathrm{kpc})\end{array}$} & \multirow[b]{2}{*}{ ОвJестS } & \multirow[b]{2}{*}{ REFERENCE } \\
\hline & Young & Intermediate & Old & & & \\
\hline \multirow{2}{*}{$d[\mathrm{~N} / \mathrm{H}] / d R \ldots \ldots$} & $-0.026 \pm 0.009$ & $\ldots$ & $\ldots$ & $6-16$ & B stars & 1 \\
\hline & $-0.007 \pm 0.013$ & $\ldots$ & $\ldots$ & $6-16$ & $\mathrm{H}$ II & 1 \\
\hline \multirow[t]{4}{*}{$d[\mathrm{O} / \mathrm{H}] / d R \ldots .}$. & $0.000 \pm 0.009$ & $\ldots$ & $\ldots$ & $6-16$ & B stars & 1 \\
\hline & $-0.045 \pm 0.022$ & $\ldots$ & $\ldots$ & $6-16$ & $\mathrm{H}$ II & 1 \\
\hline & $-0.052 \pm 0.008$ & $\ldots$ & $\ldots$ & $6-17$ & $\mathrm{H}$ II & 2 \\
\hline & & $-0.069 \pm 0.006$ & $\ldots$ & $5-11.5$ & Type II PN & 3 \\
\hline \multirow[t]{8}{*}{$d[\mathrm{Fe} / \mathrm{H}] / d R \ldots \ldots$} & $-0.089 \pm 0.025$ & & & $6.5-11$ & Open clusters & 4 \\
\hline & $\ldots$ & $-0.072 \pm 0.020$ & $-0.115 \pm 0.037$ & $7.3-14$ & Open clusters & 5 \\
\hline & $\ldots$ & $-0.023 \pm 0.059$ & $\ldots$ & $6.5-9$ & Open clusters & 4,5 \\
\hline & $\ldots$ & $-0.083 \pm 0.027$ & $\ldots$ & $9-14$ & Open clusters & 4,5 \\
\hline & $\ldots$ & $-0.072 \pm 0.054$ & $-0.149 \pm 0.064$ & $4-11$ & $\mathrm{~F}$ and $\mathrm{G}$ stars & 6 \\
\hline & $\ldots$ & $0.00 \pm 0.02$ & $0.00 \pm 0.02$ & $<8$ & F stars & 7 \\
\hline & $\ldots$ & $0.00 \pm 0.02$ & $\sim-0.15$ & $>8$ & F stars & 7 \\
\hline & $\ldots$ & $\ldots$ & $-0.261 \pm 0.102$ & $2-6$ & Gobular clusters & 8 \\
\hline
\end{tabular}

NoTE.-The radial gradients are in dex $\mathrm{kpc}^{-1}$.

${ }^{a}$ Young corresponds to age $\tau<1 \mathrm{Gyr}$, intermediate to $\tau<3$, and old to $3<\tau$.

REFERENCES.- (1) Kaufer et al. 1994; (2) Shaver et al. 1983 and Vílchez \& Esteban 1996; (3) Maciel \& Köppen 1994; (4) Panagia \& Tosi 1981 and Cameron 1985; (5) Friel 1995; (6) Edvardsson et al. 1993; (7) Jønch-Sørensen 1995; (8) Zinn 1985.

For $\tau>3$, the situation is a little more varied since open clusters and Edvardsson et al. stars yield quite similar results, around -0.115 and -0.150 , respectively. JønchSørensen distinguishes the stars according to the radial distance, obtaining roughly 0.00 and $-0.150 \mathrm{dex} \mathrm{kpc}^{-1}$, respectively, for the inner and outer Galaxy. From the globular clusters of Zinn, after the kinematic selection, we estimate about -0.260 for $R>2 \mathrm{kpc}$.

Given the large incertitudes associated with existing data and the possible selection effects involved in their interpretation, it would be incorrect to derive the conclusion of an observational evidence for a flattening of abundance gradients with time. In any event, the present status of the data allows us to say that the situation is rather evolved from how it looked until recently: now the flattening of gradients with time looks compatible with data while, vice versa, their steepening is more difficult to reconcile with them. In particular, the results from Kaufer et al. and Jønch-Sørensen raise severe doubts about the traditional interpretation of gradients' becoming stronger with time.

\section{THEORETICAL MODELS}

Because of the difficulty of finding observational data useful to discriminate whether the past radial abundances were flatter or steeper than the present ones, we now analyze the information obtained from theoretical models in order to see if some reasonable hints about their behaviors can be gained.

\subsection{Multiphase Model Description and Input Data}

We have used the theoretical models computed in FMPD and MFD for the Milky Way and six other external galaxies. These models are based on the multiphase model of Ferrini et al. (1992) and have already been described in detail in Shore \& Ferrini (1995). Only a brief outline is given here.

We assume that the primordial gas of the spheroidal protogalaxy collapses onto the galactic plane and forms out the disk. This sphere is divided into concentric cylindrical regions $1 \mathrm{kpc}$ wide, with a height defined by the galactic radius and the corresponding galactocentric distance on the disk for every region. The model is used to calculate the time evolution of the halo and disk components of every cylindrical region; the halo gas falls onto the galactic plane at a rate inversely proportional to the collapse time, which is an input parameter of the model.

In the various galactic regions, the evolution scheme considers that the diffuse gas forms molecular clouds; stars are formed by cloud-cloud collisions or by the interaction of massive stars with clouds. The rates for these processes are functions of the galactocentric radius (see FMPD) and proportional to the corresponding efficiencies representative of the probabilities of cloud formation, cloud-cloud collision, and interaction of massive stars and clouds, respectively. These efficiencies are allowed to change from galaxy to galaxy.

In several aspects the multiphase model is different from the approach adopted by other authors; it is a selfconsistent model. The star formation process is in principle nonlinear, and hence the adoption of a linear relationship between the SFR and the gas density does not seem to be appropriate: it is dangerous to linearize intrinsically nonlinear processes (see, e.g., Köppen 1994). Furthermore, the observational evidence from the solar neighborhood tells us that no star has been seen to be born from diffuse gas, but instead all direct and indirect evidence of star formation shows that stars form out of molecular clouds.

The disk is a secondary structure, generally believed to be younger than the halo and thick disk populations, so it results rather naturally to consider that the material forming the disk has been at least partially recycled into these older population stars and may only have a faint memory of the metal-free gas that in principle formed the galaxy. In other words, the characteristic infall gas requested by one-zone models, even in those simple approaches, cannot be considered metal-free, as it often is in the literature, but its metal content is tuned by the formation and evolution of stars in the halo.

It is possible to introduce radial flows, diffusion terms, and other interactions within different zones (Shore 1983; Shore \& Ferrini 1995), but since our aim is to investigate the 
simplest mechanisms of the formation and evolution of radial abundance distributions, we do not at present introduce these terms in the formalism. Moreover, Götz \& Köppen (1992) have already established that radial flows may only change a given radial gradient but they alone cannot produce it.

The inputs for the models are the present total mass radial distribution (obtained from observed rotation curves), the timescale for the collapse of gas on the galaxy disk, and the efficiencies for molecular cloud and star formation processes.

\subsection{Model Results}

The outputs of the model have been compared to observations for the galaxies studied in MFD, and the fits can be considered reasonably good. We shall consider here only the aspect of the time variation of selected radial distributions. For each galaxy, NGC 300, NGC 598, NGC 6946, NGC 3198, NGC 628, the Milky Way, and NGC 224-in order of decreasing morphological type $T$-we plot (1) the radial distribution of surface density of atomic (diffuse) gas, $\sigma_{\mathrm{H} \mathrm{II}} ;$ (2) the radial distribution of surface density of molecular gas, $\sigma_{\mathrm{H}_{2}} ;(3)$ the radial distribution of oxygen as $12+\log$ $(\mathrm{O} / \mathrm{H}) ;(4)$ the "metallicity" $[\mathrm{Fe} / \mathrm{H}]$ for three regions of the disk; and (5) the history of the SFR.

In Figure 1, we plot the radial atomic gas distributions, at different times from 2.5 to $13 \mathrm{Gyr}$. The distributions present a maximum, located in the inner part of the disk, which moves outward as time increases. In NGC 224, of an early morphological type, the evolution has proceeded more rapidly, and the maximum at $13 \mathrm{Gyr}$ is located at $12 \mathrm{kpc}$ from the center, with a shift of $5 \mathrm{kpc}$ from $2.5 \mathrm{Gyr}$. In the case of NGC 300, smaller and of a later type, the peak is located at $2 \mathrm{kpc}$, with a drift of $1 \mathrm{kpc}$ from $2.5 \mathrm{Gyr}$. The other galaxies present an intermediate behavior. They have intermediate masses between the two and belong to intermediate morphological types.

In Figure 2, we show the radial molecular gas distributions. At early evolutionary phases, their behavior can be described by an exponential function. The distributions flatten with time and decrease exponentially from a given radius for each galaxy. A maximum appears in almost all the distributions, and its time evolution is similar to that of atomic gas: the location of the maximum drifts toward larger radii at later times. Most of the molecular mass is bound between two radii, and therefore we can speak of a molecular ring.

When comparing the model results with observational data, it is evident that the models reproduce adequately the observations in the case of the atomic gas, while the fit is not as good for the molecular gas. However, it should be remembered that in the determination of the molecular hydrogen densities from $\mathrm{CO}$ luminosities, there is a large margin of uncertainty (see discussion in $\S 2$ ).

From the common behavior with time of the gaseous distributions, we may deduce that at early times there had been a vigorous activity of cloud and star formation in the inner regions, which consumed most of the material, turning it into stars and remnants. Later, the center of activity moved outward, where the reservoir of gas had not yet been depleted. In these regions, the low initial activity, plus the continuous accretion of material from the halo, allows only a moderate amount of gas to be transformed into clouds and stars.
In Figure 3, we plot the oxygen abundance distributions as a function of time for external galaxies. In most cases the computed present distributions cannot be approximated by a single straight line since they flatten in the inner regions. Further support for this behavior comes from McWilliam \& Rich (1994), who claim from a study of K giant stars in the bulge and inner disk regions that $[\mathrm{Fe} / \mathrm{H}]$ among these stars has a flat radial distribution within the solar circle and then declines beyond this distance. We remember that the multiphase model successfully reproduces the observations concerning the Galactic bulge (Mollá \& Ferrini 1995), at variance with other traditional chemical evolution models, which fail (e.g., Matteucci \& Brocato 1991).

Regarding the time evolution, different regions of the disk seem to evolve differently. The inner regions evolve more rapidly, reaching at $2.5 \mathrm{Gyr}$ abundances close to the present ones. Therefore the radial abundance distributions are much steeper at early times and flatten as galaxies age. The degree of this flattening is also different from galaxy to galaxy, being more pronounced for early types.

Figure 4 shows the model results for the Milky Way for the oxygen abundance (Fig. 4a) and for the iron abundance relative to the Sun (Fig. $4 b$ ). The calculated evolutions are the dashed lines, and the corresponding times label the curves. The boxes refer to the observational distributions from Table 1; the numbers are the corresponding references. The flattening of gradients with time is evident both from the models and the observations. The correspondence of the two is rather good; in particular, for oxygen the solution for $13 \mathrm{Gyr}$ is well inside the $\mathrm{H}$ in box. The evolution for $10 \mathrm{Gyr}$ (not shown, for simplicity) falls exactly in the PN range. For iron the early-time solution corresponds to the globular clusters, while the solutions in the range 8.5-13 Gyr fall well within the open cluster distributions, which present rather large errors anyway .

The flattening reflects a different age-metallicity relation for each region of the disk. This is illustrated in Figure 5, where we plot $[\mathrm{Fe} / \mathrm{H}]$ versus time for three regions in each galaxy. These regions are selected as those located at galactocentric distances $R=R_{\mathrm{eq}} / 1.5 \mathrm{kpc}$ for inner (I) regions, at galactocentric distances $R=R_{\mathrm{eq}} \mathrm{kpc}$ for central (C) regions, and at galactocentric distances $R=2.5 R_{\text {eff }} \mathrm{kpc}$ for outer (O) regions (where $R_{\text {eff }}$ is the effective radius and $R_{\text {eq }}$ is the radius equivalent to the solar radius for every galaxy, calculated with the expression $\left.R_{\text {eq }}=R_{\text {eff }} / R_{\text {eff, }, \mathrm{MWG}} R_{\odot}\right)$. At smaller galactocentric distances, the enrichment is much more efficient in the first few Gyr, while further away the abundance reached at each time is lower, as are the rates of increase. In the inner regions, the tendency to reach a saturation level is evident in some cases, such as NGC 628 and the Milky Way. In the case of NGC 224, $[\mathrm{Fe} / \mathrm{H}]$ even decreases after $\sim 7$ Gyr. Gas restitution by low-mass stars is dominant at those epochs in these kinds of galaxies, where the star formation rate has exhausted the gas reservoir. This material expelled from low-mass stars is less enriched than the material coming from massive stars and results in a net dilution of the amount of iron present in the region and hence a decrease of the abundance.

The importance of the morphological type or, better, of the arm class, as we underlined in previous papers, is again present and evident, and its importance is different according to the radial regions of interest.

It is worthwhile to note that the outer regions all present very similar behaviors; indeed, once normalized to a 

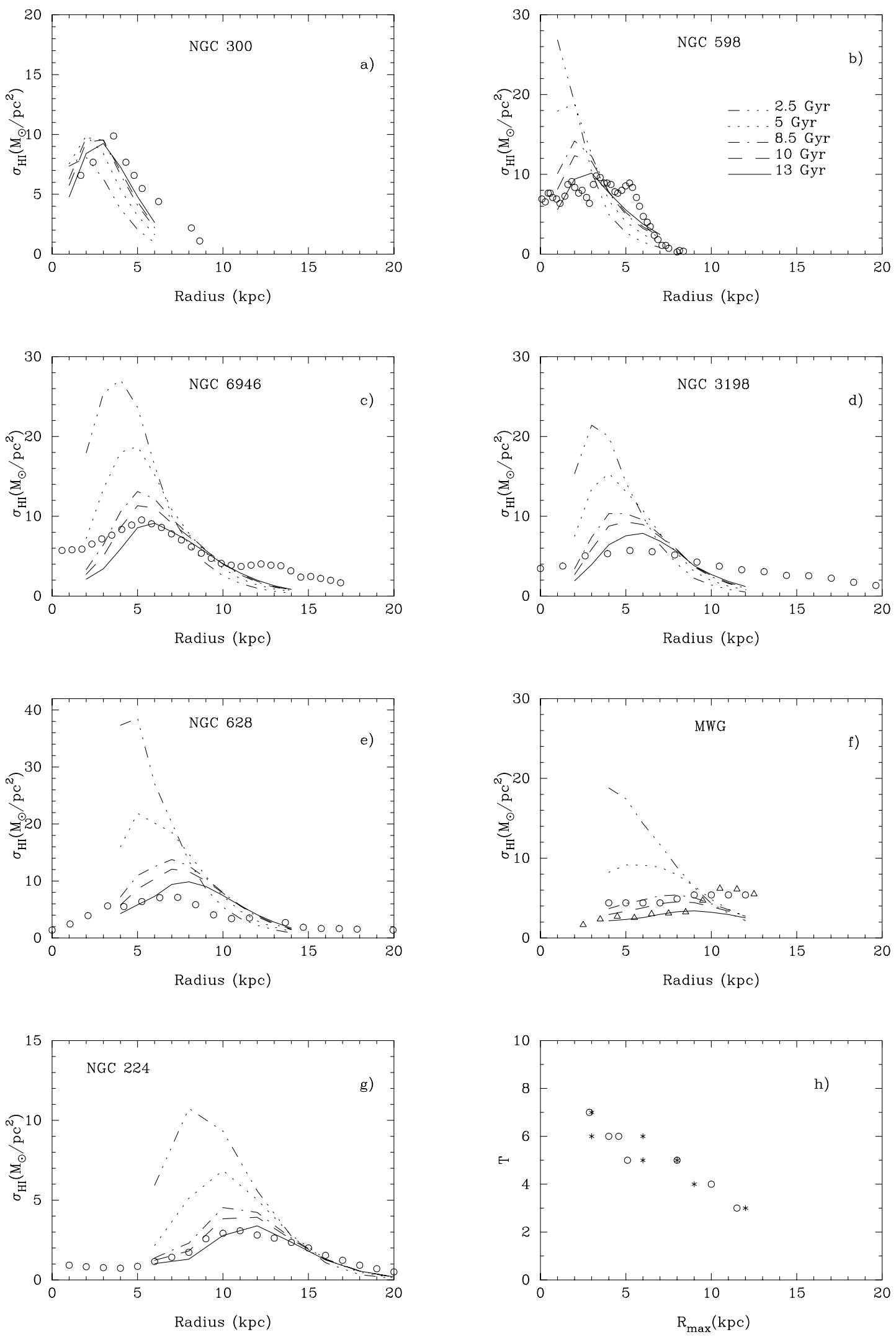

FIG. 1.-Atomic gas radial distributions obtained for different times, $2.5 \mathrm{Gyr}$ (triple-dot-dashed line), $5 \mathrm{Gyr}$ (dotted line), $8.5 \mathrm{Gyr}$ (dot-dashed line), $10 \mathrm{Gyr}$ (dashed line), and $13 \mathrm{Gyr}$ (solid line), for our galaxy sample: (a) NGC 300, (b) NGC 598, (c) NGC 6946, (d) NGC 3198, $(e)$ NGC 628, $(f)$ the Milky Way, and (g) NGC 224. In all cases data symbols refers to present observations. (When two symbols are shown, the data refer to two sets of data from different authors - see table of references for data in MFD). The type $T$ vs. the radius of the maximum in these distributions is shown in ( $h$ ) (circles are observations and asterisks are calculations). 

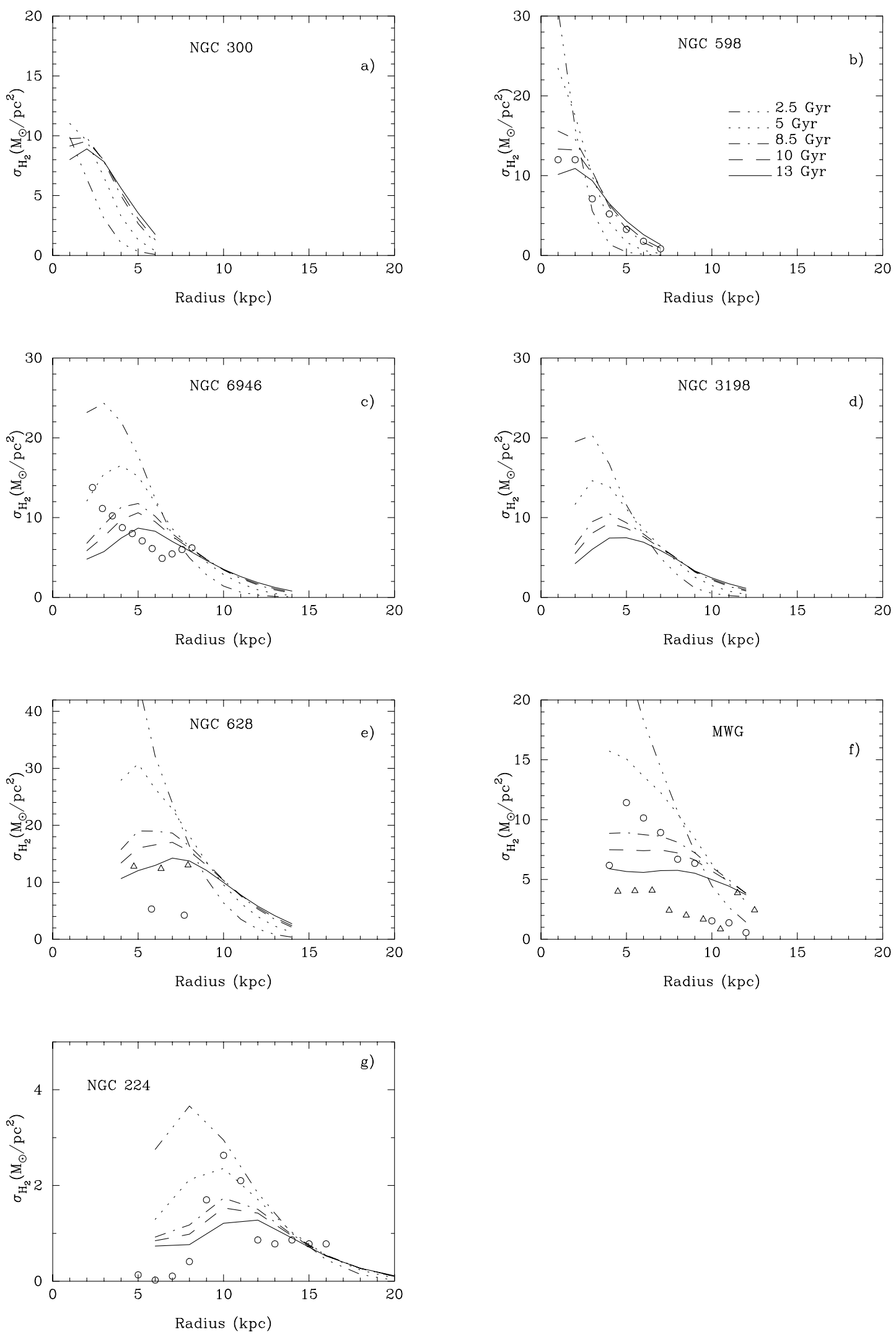

Fig. 2.-Molecular gas radial distributions for the same times and galaxies as in Fig. 1; symbols and lines have the same meanings

common value, the curves for the AMR could be superposed with a small spread among them. This is actually a prediction of the model: spiral galaxies should be very similar in their external regions, independently from their type, although the definition of external is not the same for all of them. The self-regulating nature of galactic evolution, well described by our nonlinear model, determines the rate of evolution in the different regions, according to the inter- 

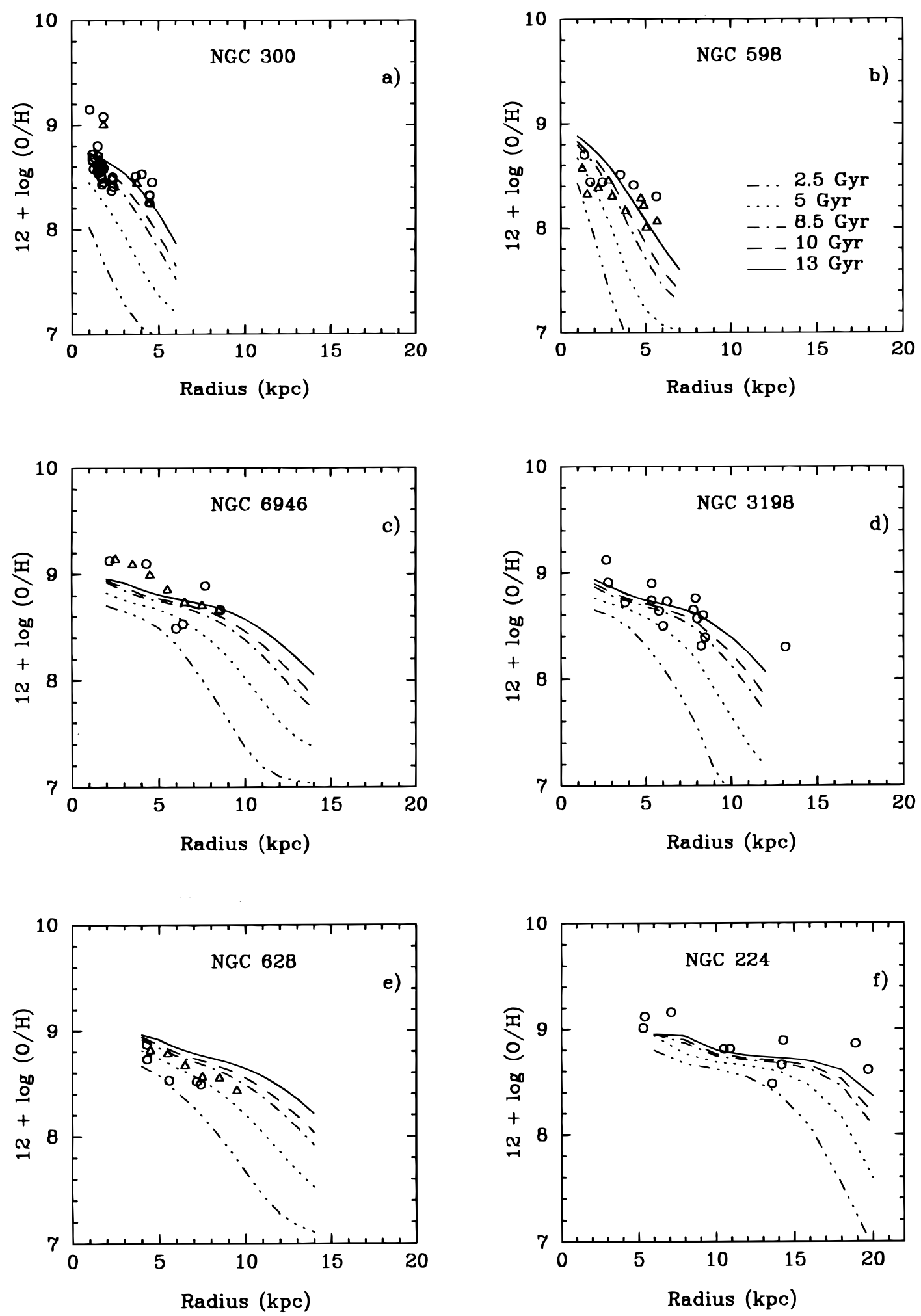

FIG. 3.-Radial distributions of oxygen abundance for the same times and galaxies as in Fig. 1, except for the Milky Way; symbols and lines have the same meanings.

actions between phases. The reduction of the efficiencies of cloud formation from diffuse gas and of star formation via cloud-cloud collisions, as well as the lower infall of matter from the halo, determine the anemic behavior of the external regions, which look very similar.

The evolution rate in each galaxy is described by the star formation rate for the solar equivalent radius, $R_{\text {eq }}$, as a function of time (Fig. 6). The model fulfills the expectations: an early-type galaxy reaches a high star formation rate at early times (NGC 224) while later types present delayed and less intense main star formation episodes.

For what concerns the different zones in each galaxy, it appears that the activity is reduced in the central parts and increased in the external ones. In Figure 7, we plot the 

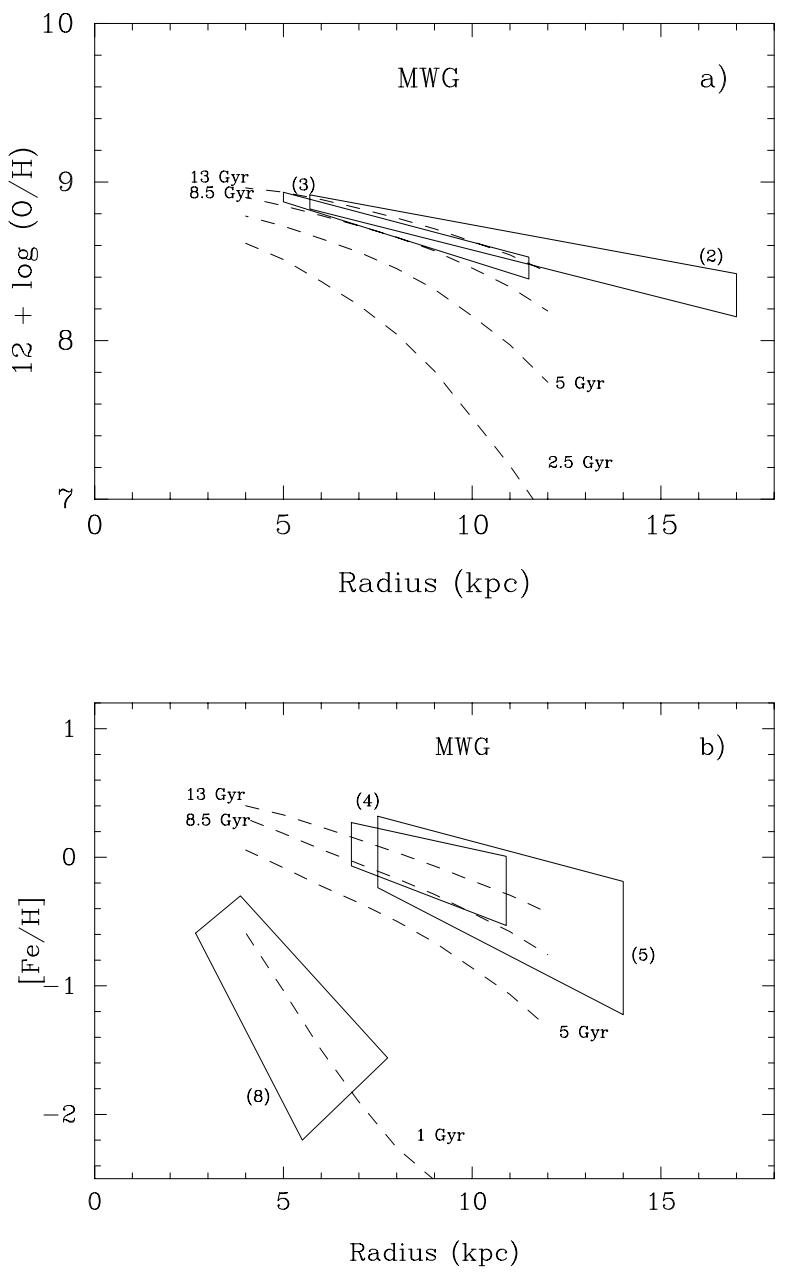

FIG. 4.-Radial distributions for the Milky Way. (a) Oxygen; boxes refer to $\mathrm{H}$ II regions and to type II PNs. (b) Iron; boxes refer to globular clusters and young and old open clusters.

radius of the region of the dominant SFR versus time for the galaxy NGC 3198 to show how this region shifts outward: it looks like a star formation wave propagating from the inner to the outer regions. Different evolutionary phases correspond to different regions involved in the star formation process. These predictions are not completely new, since Larson (1976), with a different approach, first suggested that a wave of star formation moved outward in radius with increasing time in disk galaxies. It is worthwhile to mention that the best model in Larson's approach is the one that simulates the effect of cloud interactions in the step toward star formation and also that Larson was in favor of a multicomponent model.

The model results, as it was shown in MFD, reproduce well the observations concerning the galaxies of interest and, moreover, respect the expectations concerning the different appearances of different type galaxies. For example, although data for the SFR in NGC 224 (M31) are sparse, the metallicity study of its globular cluster system (Huchra et al. 1991) and photometric studies (Bica et al. 1992; Jablonka, Alloin, \& Bica 1992) point to this galaxy's having a halo more metal rich than ours. The old disk, or thick disk, reaches greater radial distances, and it is also more metal rich. It is possible to estimate the present star formation rate at $0.5-1.3 M_{\odot} \mathrm{yr}^{-1}$, smaller than the solar value.
All this seems to indicate a more evolved state and an SFR that has been larger in the past.

On the contrary, NGC 598 (M33) has a smaller halo, less rich in metals (Schommer et al. 1993), and only the populations of internal disk and bulge can reach the solar abundances. The recent data from Minniti, Olszewski, \& Rieke (1994) give three points for a possible AMR in the most internal region, in agreement with our model results (see Fig. $5 b$ ); they conclude that this galaxy had a recent episode of star formation, of the kind that appears in Figure 6.

\subsection{The Origin of Our Results and Comparison with Other Models}

We analyze in the following a few aspects of the model with the aim of understanding the nature of the results.

The system behaves as if a threshold density for the star formation would be present in two aspects: (1) the main star formation episode is located at different radii as time varies, as if a critical condition had been achieved locally, and (2) certain regions activate only at certain times, when a critical condition is reached. The collapse time of later-type galaxies indeed contributes to this threshold behavior, since the fueling of material is less efficient.

The resulting star formation versus gas density relation follows a nonlinear law, a Schmidt law with a power index $n \neq 1$ with a radially dependent proportionality factor. (See Fig. 13 in MFD, where the SFR surface density is plotted versus the total gas surface density for the three regions defined as "inner," "middle" or "central," and "outer" for every galaxy. The power index variation is also shown in the same figure.) Note that the power law with varying exponent is a consequence of the model, not an assumption.

The nonlinearity of the star formation process is directly considered in the multiphase model, which also shows an SFR higher in the past for early-type galaxies (Ferrini \& Galli 1988), indeed one of reasons why they display higher abundances.

The growth of abundances is understandable from the star formation history: the fact that a maximum level is present in the inner regions is clearly due to the halt in the star formation as a consequence of the rapid exhaustion of the gas reservoir. In our models, a possible reason for the existence of gradients is the radial variation of the SFR. This variation is due first to the radial dependence of the model parameters when volumetric effects are introduced and to the radial dependence assumed for the infall gas rate (or for its inverse, the collapse timescale) and, second, to the radial dependence of the efficiency in forming molecular clouds from diffuse gas, included in the model to take into account the spiral wave passage, more frequent in the inner regions than in the outer ones. If the spiral wave were not present, the radial gradient would not appear or would be flatter; in fact, Mollá (1993), assuming a constant efficiency for the molecular cloud formation, found a much flatter gradient.

The models predict a steeper present-day gradient for late-type galaxies as a consequence of their smaller efficiencies and slower collapses. The computed abundance gradients flatten with time (see Table 2) as a consequence of the evolution of the galaxy. If a given galaxy is in an early evolutionary state (as occurs with late morphological type galaxies), the gradient is still steep. The gradient seems to be a differential effect between regions that evolve at a different rate, reaching their maximum levels at different times. The 

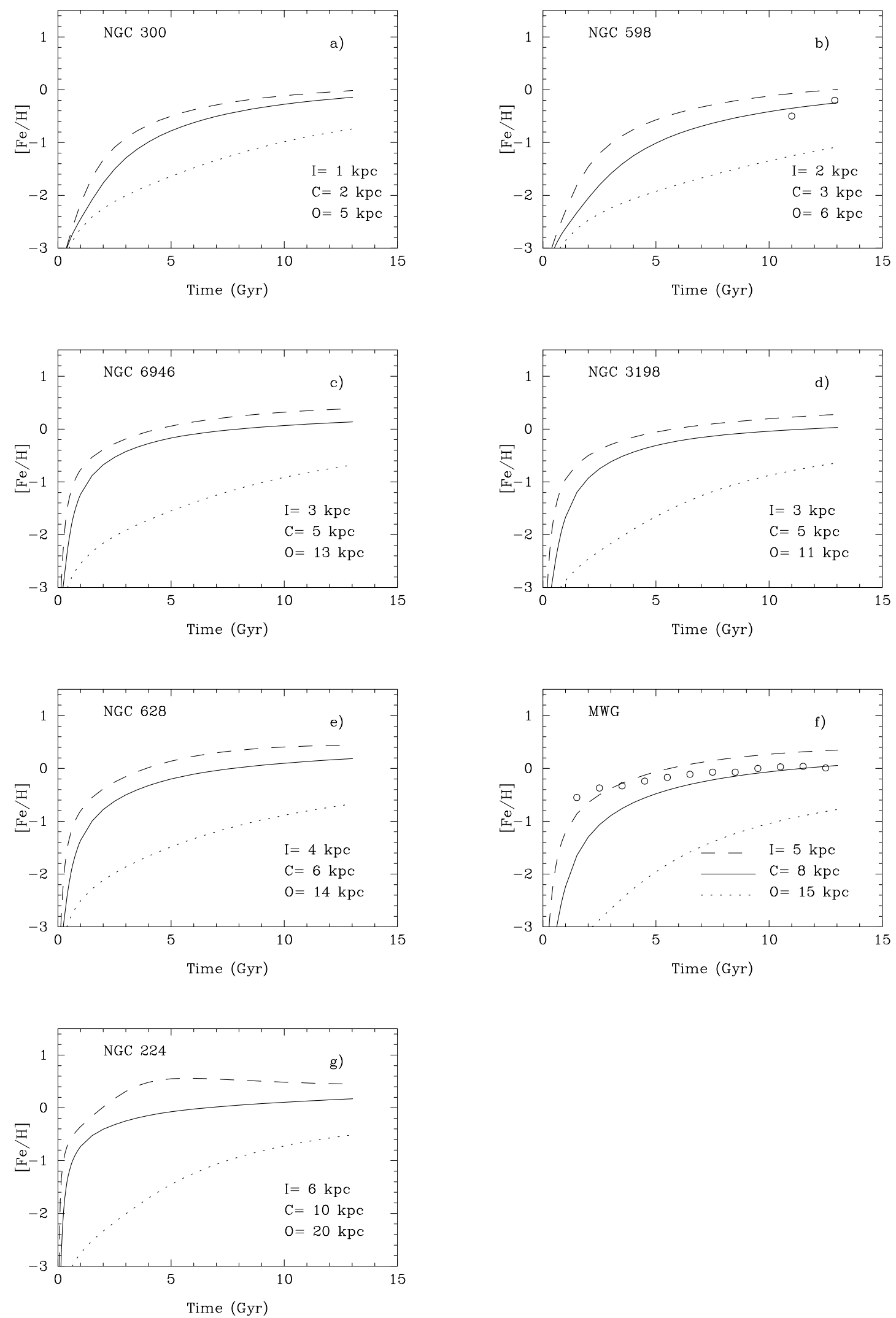

FIG. 5.-AMR for three regions, inner (I) (dashed line), central (C) (solid line), and outer (O) (dotted line), for each galaxy: (a) NGC 300, (b) NGC 598, (c) NGC 6946, $(d)$ NGC 3198, $(e)$ NGC 628, $(f)$ the Milky Way, and $(g)$ NGC 224. Data are present only for NGC 598 and for the Milky Way.

inner regions of the disks reach this level in a short time, and a steep gradient is promptly developed. When the outer regions also reach their maximum abundance level, the gradient becomes shallower and even disappears in some cases.
The earlier morphological type galaxies usually are also more massive and therefore collapse much more rapidly, showing a faster evolution and a higher SFR. Because of this, a saturation level is reached rapidly. 


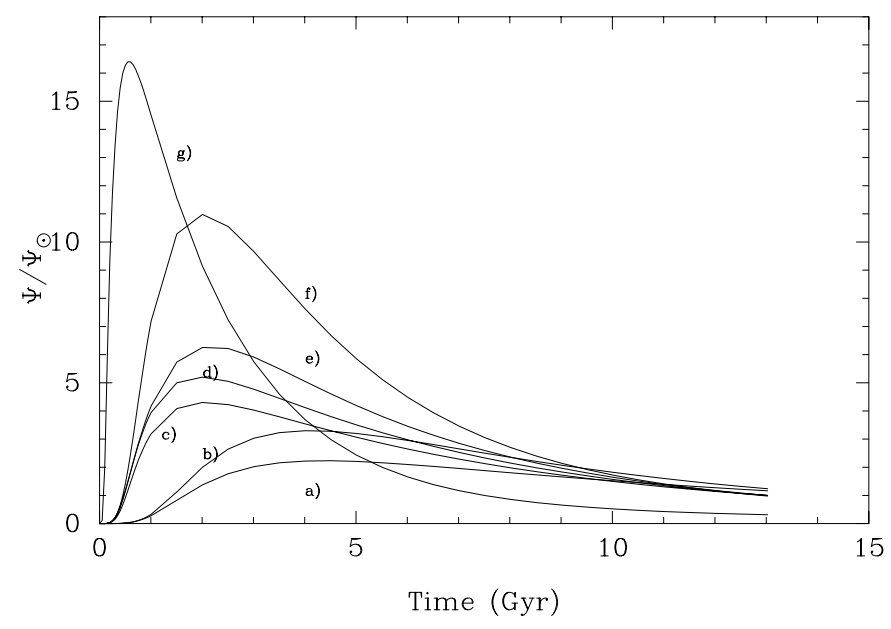

Fig. 6.-The SFR, $\Psi$, at a fixed radius $\left(R_{\text {eq }}\right)$, normalized to the present $\Psi$ at the solar radius in the Milky Way, as a function of time, for our galaxies: NGC 300 (a), NGC 598 (b), NGC 6946 (c), NGC 3198 (d), NGC 628 (e), the Milky Way (f), and NGC 224 (g) (in this case we divided $\Psi$ by 3 ). We assume a disk age of $13 \mathrm{Gyr}$ for all galaxies.

The behavior that we find numerically, and as we have shown can be understood with qualitative arguments, is opposite to the results derived numerically from most chemical evolution models, as stated in the review of the subject by Tosi (1996). Only a few papers have treated the appearance of radial abundance distributions in external galaxies (see MFD for references), most authors being more interested in fitting the Galactic distributions than in studying the general problem of abundance gradients. An even more restricted number of authors discuss explicitly the time variation of elemental distributions. Therefore the comparison is limited and not always easy to do.

The nonlinear SFR-gas density relation and/or a radial dependence of its proportionality factor are two reasons that can lead to the development of an abundance gradient, as is also found by analytical methods (Götz \& Köppen 1992). Recently, these two possibilities have been explored by Köppen (1994), using a simplified evolutionary model in order to study the time evolution of abundance gradients in both cases. The hypothesis of an SFR proportional to the gas density leads naturally to a gradient initially null and

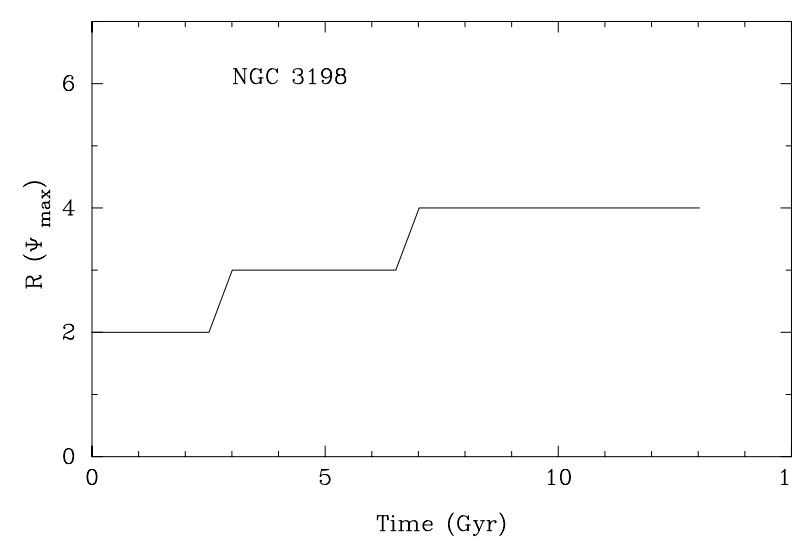

FIG. 7.- Location of the SFR $\Psi$ maximum for NGC 3198 as a function of time.
TABLE 2

COMPuted Abundance Gradients

\begin{tabular}{|c|c|c|c|c|c|}
\hline $\begin{array}{l}\text { Time } \\
\text { (Gyr) }\end{array}$ & $\frac{d[\mathrm{O} / \mathrm{H}]}{d R}$ & $\frac{d[\mathrm{~N} / \mathrm{H}]}{d R}$ & $\frac{d[\mathrm{~S} / \mathrm{H}]}{d R}$ & $\frac{d[\mathrm{C} / \mathrm{H}]}{d R}$ & $\frac{d[\mathrm{Fe} / \mathrm{H}]}{d R}$ \\
\hline \multicolumn{6}{|c|}{ NGC $300(T=7):^{a}$} \\
\hline $2.5 \ldots \ldots$ & -0.198 & -0.232 & -0.199 & -0.100 & -0.203 \\
\hline $5.0 \ldots \ldots$ & -0.198 & -0.262 & -0.200 & -0.201 & -0.211 \\
\hline $8.5 \ldots \ldots$ & -0.193 & -0.284 & -0.198 & -0.195 & -0.213 \\
\hline $10 \ldots \ldots$ & -0.190 & -0.289 & -0.195 & -0.192 & -0.212 \\
\hline $13 \ldots \ldots$ & -0.183 & -0.294 & -0.190 & -0.185 & -0.208 \\
\hline \multicolumn{6}{|c|}{ NGC $598(T=6)$ : } \\
\hline $2.5 \ldots \ldots$ & -0.258 & -0.311 & -0.259 & -0.202 & -0.266 \\
\hline $5.0 \ldots \ldots$ & -0.243 & -0.330 & -0.247 & -0.247 & -0.264 \\
\hline $8.5 \ldots \ldots$ & -0.227 & -0.341 & -0.234 & -0.230 & -0.257 \\
\hline 10 . & -0.220 & -0.342 & -0.229 & -0.223 & -0.252 \\
\hline 13. & -0.209 & -0.340 & -0.218 & -0.211 & -0.242 \\
\hline \multicolumn{6}{|c|}{ NGC $6946(T=6)$ : } \\
\hline $2.5 \ldots \ldots$ & -0.137 & -0.188 & -0.139 & -0.142 & -0.153 \\
\hline $5.0 \ldots \ldots$ & -0.119 & -0.183 & -0.124 & -0.123 & -0.144 \\
\hline $8.5 \ldots \ldots$ & -0.102 & -0.172 & -0.108 & -0.104 & -0.127 \\
\hline $10 \ldots \ldots$ & -0.096 & -0.167 & -0.102 & -0.098 & -0.121 \\
\hline 13 . & -0.085 & -0.156 & -0.093 & -0.087 & -0.109 \\
\hline \multicolumn{6}{|c|}{ NGC $3198(T=5)$ : } \\
\hline $2.5 \ldots \ldots$ & -0.222 & -0.305 & -0.228 & -0.226 & -0.238 \\
\hline $5.0 \ldots \ldots$ & -0.163 & -0.260 & -0.168 & -0.169 & -0.197 \\
\hline $8.5 \ldots \ldots$ & -0.110 & -0.204 & -0.113 & -0.119 & -0.150 \\
\hline $10 \ldots \ldots$ & -0.096 & -0.187 & -0.099 & -0.106 & -0.136 \\
\hline $13 \ldots \ldots$ & -0.078 & -0.161 & -0.079 & -0.088 & -0.115 \\
\hline \multicolumn{6}{|c|}{ NGC $628(T=5)$ : } \\
\hline $2.5 \ldots \ldots$ & -0.127 & -0.169 & -0.129 & -0.131 & -0.139 \\
\hline $5.0 \ldots \ldots$ & -0.116 & -0.172 & -0.119 & -0.119 & -0.135 \\
\hline $8.5 \ldots \ldots$ & -0.103 & -0.167 & -0.108 & -0.105 & -0.124 \\
\hline 10. & -0.098 & -0.164 & -0.104 & -0.100 & -0.120 \\
\hline $13 \ldots \ldots$ & -0.090 & -0.158 & -0.096 & -0.092 & -0.111 \\
\hline \multicolumn{6}{|c|}{ Milky Way $(T=4)$ : } \\
\hline $2.5 \ldots \ldots$ & -0.217 & -0.308 & -0.228 & -0.221 & -0.241 \\
\hline $5.0 \ldots \ldots$ & -0.127 & -0.222 & -0.133 & -0.135 & -0.168 \\
\hline $8.5 \ldots \ldots$ & -0.087 & -0.171 & -0.089 & -0.099 & -0.129 \\
\hline $10 \ldots \ldots$ & -0.079 & -0.158 & -0.081 & -0.087 & -0.119 \\
\hline 13. & -0.067 & -0.137 & -0.067 & -0.077 & -0.104 \\
\hline \multicolumn{6}{|c|}{ NGC $224(T=3)$ : } \\
\hline $2.5 \ldots \ldots$ & -0.081 & -0.148 & -0.069 & -0.063 & -0.118 \\
\hline $5.0 \ldots \ldots$ & -0.048 & -0.101 & -0.047 & -0.038 & -0.090 \\
\hline $8.5 \ldots \ldots$ & -0.032 & -0.075 & -0.037 & -0.029 & -0.065 \\
\hline $10 \ldots \ldots$ & -0.029 & -0.068 & -0.035 & -0.028 & -0.059 \\
\hline $13 \ldots \ldots$ & -0.025 & -0.059 & -0.031 & -0.025 & -0.050 \\
\hline
\end{tabular}

NoTES. - The gradients have been averaged over the radial studied range for each galaxy (see Fig. 1). They are only indicative, since there is not a unique slope in our calculated radial abundance distributions. The radial gradients are in dex $\mathrm{kpc}^{-1}$.

${ }^{\text {a }} T$ are the galactic types according to Simien \& de Vaucouleurs 1986.

steepening gradually with time, as a consequence of a continuous supply of fresh metal-free gas by infall. An SFR law that is quadratic in the gas density is much more sensitive to the initial radial distribution of gas and hence results in a steep gradient at the beginning that flattens with time. In both cases, gas motions in the disk may change the obtained distributions in a given direction, depending on the gas velocity distribution along the disk.

The results of Köppen's (1994) analysis are not new. In fact, we already verified these general aspects that do not depend strongly on the detailed model. In Mollá, Díaz, \& Tosi (1990), it was shown that the linear models produce the same results as continuous-infall models. In Mollá, Ferrini, \& Díaz (1993), the multiphase model predictions, with a nonlinear SFR and a finite infall, were compared to continuous-infall models: nonlinear SFR models produce a flattening of gradients while continuous-infall models produce gradients that steepen with time. 
The assumption of a certain amount of gas infall is common to many models, since it is needed to reproduce observational constraints such as the G dwarfs' distribution. An important question concerns the source of this infall: the galactic halo or the intergalactic medium. In the first case, we follow the classical collapse scenario for galaxy formation, used by several authors as hypothesis in their chemical evolution models (e.g., Lacey \& Fall 1985), and the infall gas will be consumed in a finite time. In the second case, the gas reservoir is supposed to last longer than the age of the galaxy, as it is due to external interactions. It is the combination of SFR and infall laws that finally determines the time evolution of gradients.

Taking into account the arguments of the previous paragraph, our results support the scenario of a finite infall of gas onto the disk, such as can be provided by a galactic halo. An unlimited source of unenriched gas seems instead to be excluded, at least for what concerns the evolution of the disk. Serendipitous accumulations from intergalactic matter or even periodic mass captures may have effects on the disk evolution, but this analysis is beyond the aims of the present paper. The halo evolution would obviously be strongly influenced by any external interaction, and the formation of globular clusters could be the clue to the matter.

Independently from the constraint, not still completely established, of the flattening of gradients with time, some well-defined behaviors in the present data can be used to discriminate among the plethora of chemical evolution models. Indeed, the evidence of a flattening in the inner Galaxy of the abundance distributions for young stellar populations (B stars and open clusters), already noted by Janes (1979) and Panagia \& Tosi (1981), appears to be a strong constraint. That suggests that we should discard traditional approaches such as that of Matteucci \& François (1989), which produced a steep gradient for late times at all radii but especially in the inner Galaxy; this disagreement with observations has already been noted by Friel (1995).

Prantzos \& Aubert (1995) compare some theoretical models in order to select the "best" one to reproduce an updated set of observational data. Although they do not treat the time evolution of gradients, they show the radial distribution of the iron abundance $[\mathrm{Fe} / \mathrm{H}]$ for stars of different ages: 2 and $4 \mathrm{Gyr}$ in their Figures 8-11e and 6 and 10 Gyr in their Figures 8-11f. From these figures, it can be seen that gradients flatten with time. Although this matter is not treated explicitly in their paper, their mathematically constructed best model is in agreement with our results. Larson (1976), although he did not explicitly discuss the point, already showed how the inner regions evolve to a saturation level.

Broadly speaking, the appearance of the gradient depends on the balance in the abundances reached at early times and how the star formation is maintained afterward. A large amount of gas, a reasonable efficiency to form clouds, and a high probability of cloud collisions lead naturally to a quick formation of a first group of stars; the massive ones will do the rest, interacting with the remaining clouds and producing further star formation in a short time (typically, their ages). A large amount of enriched material is released and mixed with the interstellar medium to create the next stellar generation. The cycle comes to an end in a relatively short time if the external fueling decreases or even stops: a large fraction of matter is locked up in low-mass stars and stellar remnants. It is hence clear what we mean by saturation of the abundances: the further release from low-mass stars is slow and rather inefficient. On the contrary, in the case of low fueling from the beginning, or of a low efficiency of instabilities to condense the gas into clouds, or of a low rate of cloud collisions, we will find a very low initial activity, with only a few formed stars. In turn, since the induced star formation processes is obviously efficient only if there are both stars and clouds largely present, the few massive stars will not succeed to stimulate adequately the nearby molecular medium, maintaining a low level of star formation. The enrichment will consequently be scarce. The process of induced star formation always acts at the same level: given enough clouds within a few parsecs around $10 \mathrm{O}, \mathrm{B}$ stars, new associations will be generated in $10^{7} \mathrm{yr}$; the process is clearly self-regulating. The formation of clouds and the formation of stars by their interactions depend instead on the environment and on the coupling with the exterior: the fall of new gas useful to form clouds will help to maintain a certain fraction of clouds. The efficiency and timescales associated with cloud formation, cloud-cloud interactions, and gas infall will determine the evolution of the galactic region. And this will be universal for all galaxies. The nature of large-scale dynamics of one particular object, depending on its mass, on the distribution of angular momentum per unit mass, on the spiral density wave, and on the particular location (e.g., halo, bulge), will influence the possible evolutions.

It may happen anyway that the inefficient consumption of clouds will lead to an accumulation of the same clouds; the spontaneous channel of star formation via cloud-cloud collisions could hence be opened abruptly, giving rise, with the help of the stimulated process, to a very intense phase of star formation-a burst. This discussion is not totally new, but is presented here for the first time concerning abundance gradients. Our approach to the comprehension of the morphological distinction of spiral galaxies is based on it (Ferrini 1987; Ferrini \& Galli 1988; for a general discussion see Shore \& Ferrini 1995).

\section{CONCLUSIONS}

1. The flattening of gradients with time results as a quite natural effect of the history of star formation: the SFR is higher where more gas, in the form of clouds, is present, i.e., in the central regions, if we follow the collapse scenario. On the other hand, the steepening of gradients is more difficult to explain given the observed flattening of the AMR. A simpleminded sketch is drawn in Figure 8. In Figure 8a, we show schematically the expected AMR in three Galactic regions in the case of gradients flattening with time; the gradient is $\sim 0.0$ for $R<1 R_{\odot}$ and decreases with time for $R>1 R_{\odot}$. In Figure $8 b$, we show how the AMR would appear for a gradient that steepens with time, more efficiently in the inner regions, as it results from other models. Maybe future data will change the situation, but the present observational status of the AMR, as discussed in $\S 2.2 .1$, seems to indicate that case $a$ has to be preferred.

2. The presence of radial abundance distributions and their time evolution seems to be a universal feature in spiral galaxies, and we provide the distinct behaviors for the different morphological types.

3. We find that the atomic gas surface density may present a maximum at a given radius; its location varies with time, and different galaxy types present different drifts in gas distribution. Since the $\mathrm{H}$ I surface density is related to 

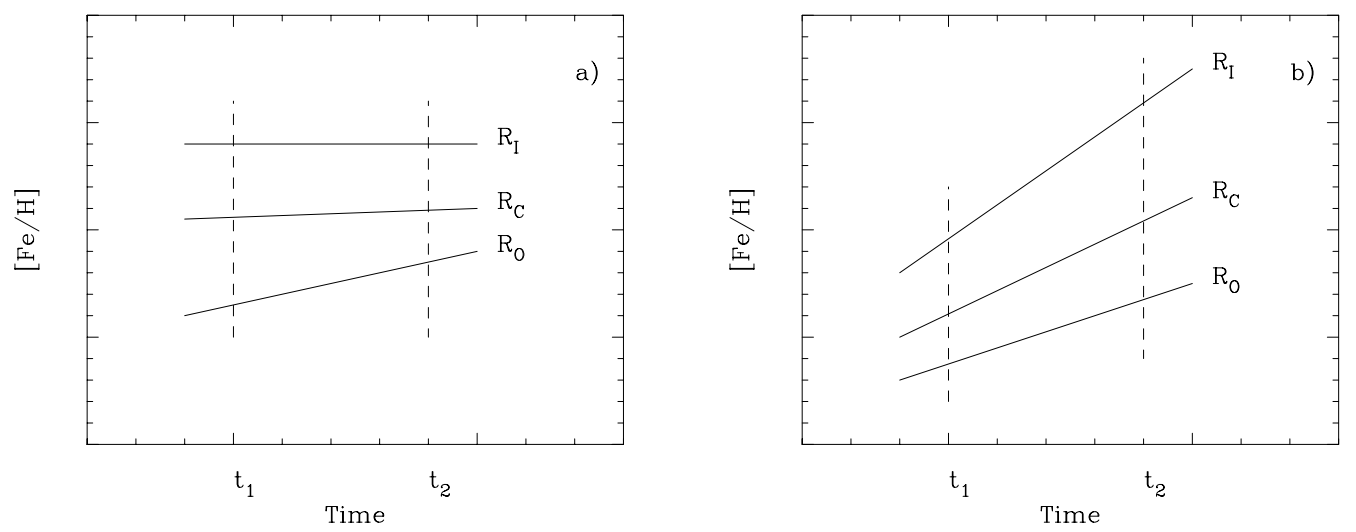

FIG. 8. - Schematic sketch of the expected AMR in the case of radial abundance gradients (a) flattening with time and $(b)$ steepening with time

the intensity of $21 \mathrm{~cm}$ line, high-resolution observations may reveal this evolution in intermediate/high-redshift objects.

4. A molecular ring is present in all our computed models. Its time and space variation are predicted.

5. The outer galactic regions have rather similar evolutionary histories, with a low level of activity lasting for long times.

6. The SFR, $\Psi$, for each galaxy, reaches asymptotically a similar value for every region within the equivalent radius.

7. We reinforce, from the last two points, our view that galaxies will present activity for times as long as the Hubble time. We remember that in Ferrini \& Galli (1988) we proposed a solution to the so-called Roberts time paradox. An initial mass function with a higher fraction of massive stars than the solar-neighborhood initial mass function is not required, as neither is a difference in the ages of disks (see Sommer-Larsen 1996). In our approach what is necessary is to account for the feedback phenomena that are present in the processes concerning star formation. The multiphase model handles them naturally.

8. The star-forming activity moves toward external regions, essentially because the inner ones more efficiently consume their fuels in shorter times. This fact is related to the preferred infall history adopted in the models. In a similar way to Larson (1976), we have chosen a finite infall, determined by the accretion of the halo gas into the forming disk.

The presence and the time evolution of radial abundance gradients may offer criteria to distinguish among chemical evolution models. Now, in the literature, there is a very large number of them; we suggest that it would be more interesting to study chemodynamical evolutionary models, rather than chemical evolution ones. Paraphrasing Larson (1976), it is important to investigate the factors that determine the SFR and the initial mass function, more than run a large amount of parametric models aimed at fitting observations. Perhaps this effort will help us make progress in the understanding of galactic evolution.

We thank an anonymous referee for a very critical reading of this paper that forced us to a radical revision, resulting in a much improved version. This work has been partially supported by Integrated Action with Italy A-28 and DGICYT project PB-93-052. Contributions from MURST and ASI are acknowledged. F. F. thanks the Universidad Autónoma de Madrid for an invitation for a sabbatical period, during which this paper was carefully revised. This research has made use of the NASA/IPAC Extragalactic Database (NED), which is operated by the Jet Propulsion Laboratory, Caltech, under contract with the National Aeronautics and Space Administration.
Alfaro, E. J., Cabrera-Caño, J., \& Delgado, A. J. 1993, ApJ, 402, L53

Alfaro, E. J., \& Delgado, A. J., eds. 1995, The Formation of the Milky Way (Cambridge: Cambridge Univ. Press)

Barry, D. C. 1988, ApJ, 334, 436

Bica, E., Jablonka, P., Santos, J. F. C., Alloin, D., \& Dottori, H. 1992, A\&A, 260, 109

Bronfmann, L., Cohen, R. S., Alvarez, H., May, J., \& Thaddeus, P. 1988, ApJ, 324, 248

Cameron, L. M. 1985, A\&A, 147, 47

Carlberg, R. G., Dawson, P. C., Hsu, T., \& VandenBerg, D. A. 1985, ApJ, 294, 674

Deul, E. R., \& den Hartog, R. H. 1990, A\&A, 229, 362

Díaz, A. I. 1989, in Evolutionary Phenomena in Galaxies, ed. J. E. Beckman \& B. E. J. Pagel (Cambridge: Cambridge Univ. Press), 377

. 1994, in Violent Star Formation from 30 Doradus to QSOs, ed. G. Tenorio-Tagle (Cambridge: Cambridge Univ. Press), 105

Edvardsson, B., Andersen, J., Gustafsson, B., Lambert, D. L., Nissen, P. E., \& Tomkin, J. 1993, A\&A, 275, 101

Ferrini, F. 1987, in Evolution of Galaxies, ed. J. Palouš (Ondrejov, Czechoslovakia: Astron. Inst. Czechoslovak Acad. Sci.), 337

. 1995, in The Formation of the Milky Way, ed. E. J. Alfaro \& A. J. Delgado (Cambridge: Cambridge Univ. Press), 191

Ferrini, F., \& Galli, D. 1988, A\&A, 195, 27

Ferrini, F., Matteucci, F., Pardi, C., \& Penco, U. 1992, ApJ, 387, 138

Ferrini, F., Mollá, M., Pardi, M. C., \& Díaz, A. I. 1994, ApJ, 427, 745 (FMPD)

Fich, M., \& Silkey, M. 1991, ApJ, 366, 107

\section{RFERENCES}

Friel, E. D. 1995, ARA\&A, 33, 381

Garwood, R. N., \& Dickey, J. M. 1989, ApJ, 338, 841

Geisler, P. 1987, AJ, 94, 84

Götz, M., \& Köppen, J. 1992, A\&A, 262, 455

Grabelsky, D. C., Cohen, R. S., Bronfmann, L., Thaddeus, P., \& May, J. 1987, ApJ, 315, 122

Grenon, M. 1988, J. Astrophys. Astron., 8, 123

Güsten, R., \& Mezger, P. G. 1982, Vistas Astron., 25, 159

Harris, H. C. 1981, AJ, 86, 719

Huchra, J. P., Brodie, J. P., \& Kent, S. M. 1991, ApJ, 370, 495

Jablonka, P., Alloin, D., \& Bica, E. 1992, A\&A, 260, 97

Janes, K. A. 1979, ApJS, 39, 135

Jønch-Sørensen, H. 1995, A\&A, 298, 799

Kaufer, A., Szeifert, T., Krenzin, R., Bashek, B., \& Wolf, B. 1994, A\&A, 289, 740

Kenney, J. 1995, in The Interstellar Medium in Galaxies, ed. J. M. van der

Hulst (Dordrecht: Kluwer), in press

Kennicutt, R. C., Jr. 1989, ApJ, 344, 685

Köppen, J. 1994, A\&A, 281, 26

Lacey, C. G., \& Fall, S. M. 1985, ApJ, 290, 154

Larson, R. B. 1976, MNRAS, 176, 31

Larson, R. B., \& Tinsley, B. M. 1978, ApJ, 219, 46

Lewis, J. R., \& Freeman, K. C. 1989, AJ, 97,139

Luck, R. E. 1982, ApJ, 256, 177

Maciel, W. J., \& Köppen, J. 1994, A\&A, 282, 436

McWilliam, A., \& Rich, R. M. 1994, ApJS, 91, 749

Mead, K. N., \& Kutner, M. L. 1988, ApJ, 330, 339 
Mead, K. N., Kutner, M. L., \& Evans, N. J. 1990, ApJ, 354, 492

Matteucci, F., \& Brocato, E. 1991, ApJ, 365, 539

Matteucci, F., \& François, P. 1989, MNRAS, 239, 885

Matteucci, F., \& Greggio, L. 1986, A\&A, 154, 279

Minniti, D. 1996, ApJ, 459, 175

Minniti, D., Olszewski, E. W., \& Rieke, M. 1994, in Third ESO/CTIO

Workshop on the Local Group, ed. A. Layden \& J. Storm (Garching: ESO), 275

Mollá, M. 1993, Ph.D. thesis, Univ. Autónoma de Madrid

Mollá, M., Díaz, A. I., \& Tosi, M. 1990, in Chemical and Dynamical Evolution of Galaxies, ed. F. Ferrini, F. Matteucci, \& J. Franco (Pisa: ETS), 577

Mollá, M., \& Ferrini, F. 1995, ApJ, 454, 726

Mollá, M., Ferrini, F., \& Díaz, A. I. 1993, in The Feedback of Chemical Evolution on the Stellar Content of Galaxies, ed. D. M. Alloin \& G. Stasińska (Meudon: Imprimerie de L'Obs. de Paris), 258 1996, ApJ, 466, 668 (MFD)

Oey, M. S., \& Kennicutt, R. C., Jr. 1993, ApJ, 411, 137

Panagia, N., \& Tosi, M. 1981, A\&A, 96, 306

Pardi, C., Ferrini, F., \& Matteucci, F. 1995, ApJ, 444, 207

Prantzos, N., \& Aubert, O. 1995, A\&A, 302, 69

Rana, N. C. 1991, ARA\&A, 29, 129

Sandage, A. 1986, A\&A, 161, 89

Sanders, D. B., Solomon, P. M., \& Scoville, N. Z. 1984, ApJ, 276, 182

Schommer, R. A., Christian, C. A., Caldwell, C. A., Bothun, G. D., \& Huchra, J. 1993, AJ, 101, 785
Schuster, W. J., \& Nissen, P. E. 1989, A\&A, 222, 69

Shaver, P. A., McGee, R. X., Newton, L. M., Banks, A. C., \& Pottash, S. R. 1983, MNRAS, 204, 53

Shore, S. N. 1983, ApJ, 265, 202

Shore, S. N., \& Ferrini, F. 1995, Fundam. Cosmic Phys., 16, 1

Simien, F., \& de Vaucouleurs, G. 1986, ApJ, 302, 564

Sodroski, T. J. 1991, ApJ, 366, 95

Sommer-Larsen, J. 1996, ApJ, 457, 118

Talbot, R. J. 1980, ApJ, 235, 821

Tosi, M. 1996, in ASP Conf. Proc. 98, From Stars to Galaxies, ed. C. Leitherer, U. Fritze-von Alvensleben, \& J. Huchra (San Francisco: ASP), 299

Tosi, M., \& Díaz, A. I. 1985, MNRAS, 217, 571

Twarog, B. A. 1980, ApJ, 242, 242

Verter, F., \& Hodge, P. 1995, ApJ, 446, 616

Vila-Costas, B., \& Edmunds, M. G. 1992, MNRAS, 259, 121

Vílchez, J. M., \& Esteban, C. 1996, MNRAS, 280, 720

Wilson, C. D. 1995, ApJ, 448, L97

Wilson, C. D., \& Scoville, N. 1991, ApJ, 370, 184

Wouterloot, J. G. A., Brand, J., Burton, W. B., \& Kwee, K. K. 1990, A\&A, 230, 21

Young, J. S. 1987, in IAU Symp. 115, Star Forming Regions, ed. M. Peimbert \& J. Jugaku (Dordrecht: Reidel), 557

Zaritsky, D., Kennicutt, R. C., Jr., \& Huchra, J. P. 1994, ApJ, 420, 87

Zinn, R. 1985, ApJ, 293, 424 